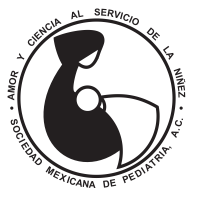

\title{
Síndrome de dificultad respiratoria aguda neonatal
}

\author{
Jorge Luis Sánchez, ${ }^{\ddagger}$ Camila Suaza-Vallejo, ${ }^{\ddagger}$ Daniel Felipe Reyes-Vega, ${ }^{\S, *}$ \\ Carlos Fonseca-Becerra ${ }^{\ddagger}, \|$ \\ ‡ Universidad Surcolombiana; ${ }^{\S}$ Hospital Universitario Hernando Moncaleano Perdomo; \\ " Hospital Universitario de Neiva. Neiva, Colombia.
}

\begin{abstract}
RESUMEN
La primera definición consenso para síndrome de dificultad respiratoria aguda (SDRA) neonatal fue establecida en 2015 a partir de un análisis de la literatura realizado por un comité de 12 expertos. En este consenso se estableció un conjunto de criterios clínicos y paraclínicos para la definición de esta condición específica y también dejó en claro algunas situaciones excluyentes comunes en los neonatos. Presentamos a un recién nacido de 27 días de vida con SDRA en el Hospital Universitario de la Ciudad de Neiva, Colombia.
\end{abstract}

Palabras clave: SDRA neonatal, criterios de inclusión, síntomas, Colombia.

\section{INTRODUCCIÓN}

El síndrome de dificultad respiratoria aguda (SDRA) neonatal es una condición médica de la cual se tenía conocimiento desde hace varios años; sin embargo, no había sido definida con parámetros clínicos y paraclínicos específicos hasta el año 2015 cuando se llevó a cabo el consenso de Montreux en Suiza. ${ }^{1}$ La primera referencia sobre el SDRA en niños se remonta a 1967 donde se presentan 12 pacientes con disnea, taquipnea y cianosis con resistencia a la oxigenoterapia, pérdida de la funcionalidad pulmonar e infiltración alveolar difusa. En autopsia se identificó atelectasia, edema alveolar y hemorragia pulmonar. ${ }^{2}$

\footnotetext{
* Correspondencia: DFRV, danfereve@hotmail.com Conflicto de intereses: Los autores declaran que no tienen. Citar como: Sánchez JL, Suaza-Vallejo C, Reyes-Vega DF, Fonseca-Becerra $\mathrm{C}$. Síndrome de dificultad respiratoria aguda neonatal. Rev Mex Pediatr. 2020; 87(3):115-118. doi: 10.35366/94843 [Acute neonatal respiratory distress syndrome]
}

\begin{abstract}
The first consensus definition for neonatal acute respiratory distress syndrome (ARDS) was established in 2015 from analysis of the literature conducted by a committee of 12 experts. This group established a set of clinical and paraclinical criteria for the definition of this specific condition and also made clear some common exclusion situations in neonates. We present a 27-day-old newborn with ARDS at the University Hospital of the City of Neiva, Colombia.
\end{abstract}

Keywords: Neonatal ARDS, inclusion criteria, symptoms, Colombia.

En la actualidad ya existe una caracterización radiológica que incluye infiltrados alveolares bilaterales, irregulares en ambos campos pulmonares. ${ }^{3}$ Adicionalmente se estableció el umbral de presión capilar pulmonar, para diferenciar el SDRA de la insuficiencia cardiaca congestiva. Las causas pueden ser enfermedades pulmonares (primarias) o no pulmonares (secundarias). ${ }^{4}$

Se ha desarrollado puntajes de lesión pulmonar; inicialmente por Murray y colaboradores, los cuales fueron modificados una década después por Newth y su equipo. ${ }^{5}$ En 1994 en la Conferencia de Consenso Estadounidense-Europeo se estableció el concepto de SDRA. ${ }^{6}$ Posteriormente, en Berlín se postularon tres clases de gravedad clínica. ${ }^{7,8}$ Sin embargo, aún no se había diseñado una definición para la población pediátrica ni para la neonatal.

Durante la Conferencia de Consenso de Lesiones Pulmonares Agudas Pediátricas (PALICC) se estableció la primera definición de SDRA en población pediátrica. ${ }^{9} \mathrm{El}$ consenso representaba un avance importante; 
sin embargo, no tenía en cuenta a los recién nacidos. Es importante resaltar que en los neonatos el retraso en el desarrollo pulmonar puede verse influenciado por enfermedades pulmonares congénitas, displasia broncopulmonar, cardiopatías congénitas, entre otras. Además, tanto la inmunidad innata como la inmunidad son diferentes, que los niños mayores. ${ }^{10}$ Estas características podrían afectar la epidemiología, el curso clínico y el pronóstico de los pacientes con SDRA. ${ }^{1}$ Por lo anterior, en 2015 el Comité Internacional en Montreux, Suiza, conformado por neonatólogos e intensivistas pediátricos de Europa, Norteamérica y Australia, proponen una serie de criterios diagnósticos clínicos y paraclínicos (Tabla 1) para la definición de SDRA neonatal, que puede ser aplicable en unidades de cuidado intensivo neonatal.

Presentamos el caso de un $\mathrm{RN}$ de 27 días de vida con un cuadro clínico concordante con la descripción de SDRA neonatal en la ciudad de Neiva, Colombia.

\section{PRESENTACIÓN DEL CASO}

$\mathrm{RN}$ masculino de 27 días de vida al ingreso, producto de embarazo normal de 38 semanas de gestación, peso al nacer 3.660 g, obtenido por parto y con adaptación neonatal espontánea. Consulta a servicio de urgencias por cuadro clínico de dos días de evolución consistente en tos productiva que inició después que la madre le administra aceite mineral; posteriormente se agregó taquipnea y dificultad respiratoria, manifestada tiraje subcostal.

A la exploración lo más evidente fue la dificultad respiratoria. Se toman exámenes de laboratorio, encontrando leucocitos de $14,200 / \mathrm{mm}^{3}$, siendo $67 \%$ de neutrófilos; hemoglobina de $12.3 \mathrm{mg} / \mathrm{dL}$, plaquetas de 374,000 y proteína C reactiva $0.29 \mathrm{mg} / \mathrm{dL}$. En la radiografía de tórax se evidencia infiltrados pulmonares difusos de predominio en pulmón derecho (Figura 1A).

Durante la evolución, el paciente persistió con deterioro respiratorio, a pesar de la administración de oxígeno y de broncodilatador, por lo que se trasladó a Unidad de Cuidados Intensivos Neonatal (UCIN). A los dos días de su llegada al hospital, en una nueva Rx de tórax (Figura 1B) se observó empeoramiento de los hallazgos iniciales, con presencia de infiltrados algodonosos bilaterales.

El panel respiratorio y hemocultivos fueron negativos. Por lo anterior, el servicio de neumología pediátrica establece el diagnóstico de neumonía lipoidea exógena, por lo que solamente se otorga el soporte ventilatorio con máscara nasal y otras medidas generales, sin antimicrobianos ni esteroides.

Por ecocardiograma, se descarta participación cardiológica (Figura 2), por lo que, aunado a acidosis

\section{Tabla 1: Definición de Montreux de síndrome de dificultad respiratoria aguda neonatal.}

\section{Periodo}

Criterios de exclusión

Imagen pulmonar

Origen del edema

Déficit de oxigenación expresado como Ol*
Inicio agudo (una semana) a partir de una alteración clínica conocida o sospecha de la misma SDR, TTN o anomalías congénitas como una afección respiratoria aguda primaria

Opacidades o infiltrados difusos, bilaterales e irregulares, u opacificación completa de los pulmones, que no se explican completamente mediante derrames locales, atelectasia, SDR, TTN o anomalías congénitas

Ausencia de cardiopatía congénita que explique el edema (esto incluye el conducto arterioso con congestión pulmonar si no existe hemorragia pulmonar aguda). La ecocardiografía es necesaria para verificar el origen del edema

$$
\begin{aligned}
& \text { SDRA leve: } 4 \leq \mathrm{Ol}<8 \\
& \text { SDRA moderado: } 8 \leq \mathrm{OI}<16 \\
& \text { SDRA severo: } \mathrm{Ol} \geq 16
\end{aligned}
$$

SDRA = síndrome de dificultad respiratoria aguda, $\mathrm{SDR}$ = síndrome de dificultad respiratoria, $\mathrm{TTN}$ = taquipnea transitoria del neonato, $\mathrm{OI}=$ índice de oxigenación. * El Ol se puede calcular mediante el uso de valores arteriales 0 , si no se dispone de valores arteriales, valores de tensión de oxígeno transcutáneos, con dispositivos transcutáneos calibrados adecuadamente. En el caso de hipertensión pulmonar persistente del neonato y ductus arterioso permeable deben utilizarse valores de $\mathrm{PaO}_{2}$ preductal.

La definición se aplica desde el nacimiento hasta las 44 semanas, la edad postmenstrual o hasta las cuatro semanas, la edad postnatal (para los neonatos nacidos después de las 40 semanas, la edad postmenstrual). Para que el síndrome sea definido se deben cumplir todos los criterios.

Ol * se debe calcular si es posible con las medidas más precisas disponibles. El síndrome se puede diagnosticar en cualquier edad gestacional o peso al nacer. Tomado y adaptado de: De Luca D, Van Kaam AH, Tingay DG, Courtney SE, Danhaive O, Carnielli VP et al. The Montreux definition of neonatal ARDS: biological and clinical background behind the description of a new entity. Lancet Respir Med. 2017; 5 (8): 657-666. 

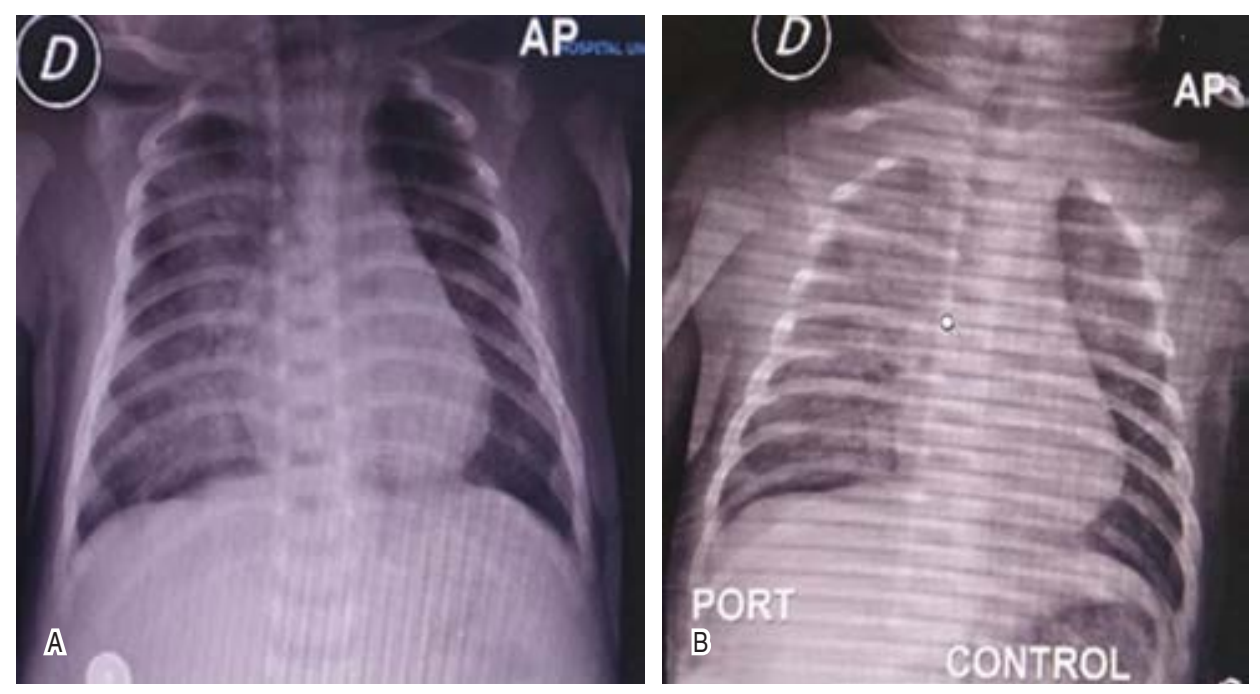

Figura 1:

A) Radiografía de tórax de ingreso. Se evidencia presencia de infiltrados pulmonares difusos de predominio en pulmón derecho. B) Radiografía de control. Aumento de radiopacidad de predominio derecho, sin observarse franca consolidación, sin pérdida de volumen pulmonar, no se observa broncograma aéreo. Sin signos de sobrecarga pulmonar. metabólica, hiperlactatemia e índice de oxigenación de 15, se estable el diagnóstico de SDRA neonatal.

Posteriormente, la evolución del paciente fue satisfactoria, con disminución de dificultad respiratoria, permitiendo destete de soporte ventilatorio no invasivo y traslado a cuidado general donde fue dado de alta a los 10 días de la hospitalización.

\section{DISCUSIÓN}

Presentamos el caso de un neonato que desarrolló SDRA secundario a la administración de aceite mineral. El primer uso del término SDRA neonatal se describió en 1989 y caracterizaba a neonatos con asfixia perinatal y meconio. ${ }^{11}$ La motivación a establecer una definición específica para neonatos fue para reconocer diferencias significativas con la población adulta, ${ }^{12}$ como el hecho de que los pulmones de un $\mathrm{RN}$ después del parto aún continúan en proceso de alveolización, tienen menor número de alvéolos, presentan una pared torácica más flexible que dificulta la adaptación de los movimientos respiratorios para una mayor oxigenación. Además, la inserción de la estructura del diafragma es diferente y su respiración tidal utiliza volúmenes más pequeños con frecuencias más altas. ${ }^{13}$

En general, el SDRA neonatal se caracteriza por disfunción cualitativa o cuantitativa de surfactante pulmonar que afecta tanto a las proteínas como a los fosfolípidos, así como a extensa afección del tejido pulmonar. ${ }^{14,15}$ Es importante tener en cuenta que en los neonatos se presenta una serie de trastornos respiratorios similares a SDRA como el síndrome de as- piración de meconio, neumonía biliar y la insuficiencia respiratoria secundaria a una corioamnionitis grave $o$ sepsis. ${ }^{16}$ También se debe entender que el deterioro del surfactante endógeno y la inflamación del intersticio pulmonar explican la respuesta limitada y a menudo transitoria a los surfactantes exógenos usados como medida terapéutica. ${ }^{17,18} \mathrm{El}$ deterioro del surfactante pulmonar se debe probablemente a la activación descontrolada de la fosfolipasa A2, la cual se desencadena con la cascada inflamatoria en el pulmón, hidrolizando

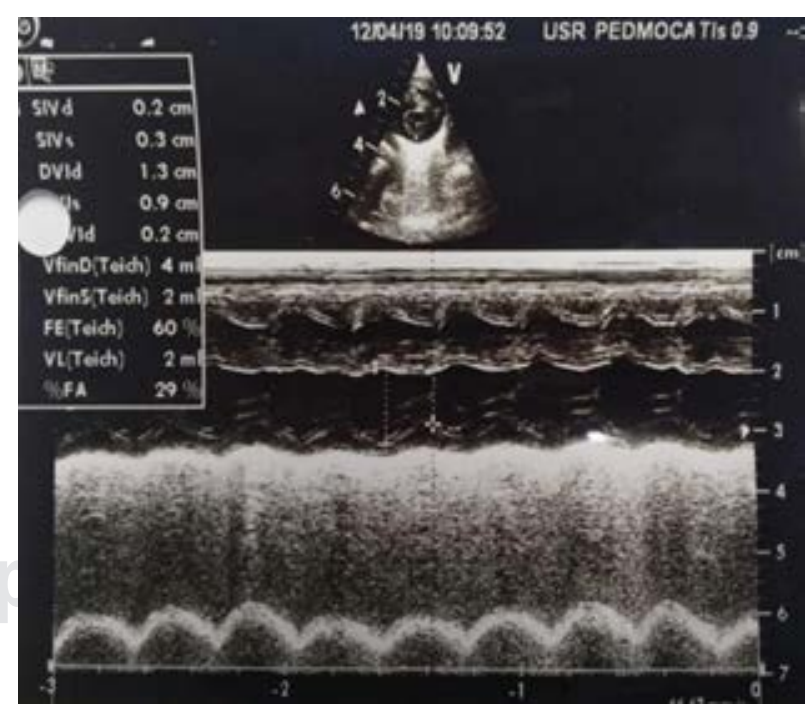

Figura 2: Ecocardiograma. Se evidencia buena función sistodiastólica ventricular izquierda, con gasto cardiaco de $250 \mathrm{~mL} /$ $\mathrm{kg} /$ minuto, sin hallazgos secundarios de hipertensión pulmonar. Ductus arterioso cerrado. 


\section{Tabla 2: Factores desencadenantes de síndrome de dificultad respiratoria aguda neonatal.}

Factores primarios (intrapulmonares)

Factores secundarios (extrapulmonares)

- Infección local

- Neumonía biliar

- Bronquiolitis

- Aspiración de meconio, sangre, agua, bilis o secreción gástrica

Tomado y adaptado de: De Luca D, Van Kaam AH, Tingay DG, Courtney SE, Danhaive O, Carnielli VP et al. The Montreux definition of neonatal ARDS: biological and clinical background behind the description of a new entity. Lancet Respir Med. 2017; 5 (8): 657-666.

los fosfolípidos de la película de surfactante, produciendo ácidos grasos libres que son sustratos para la activación de más fosfolipasa, convirtiendo el mecanismo en un círculo de retroalimentación positiva que empeora la condición del paciente. ${ }^{16}$

Los factores desencadenantes de SDRA pueden ser primarios, como neumonía, aspiración de meconio, sangre, agua, leche, bilis o secreción gástrica. Mientras que los factores secundarios o extrapulmonares pueden ser sepsis, corioamnionitis, enterocolitis necrosante o asfixia perinatal (Tabla 2). ${ }^{19}$

\section{REFERENCIAS}

1. De Luca D, Van Kaam AH, Tingay DG, Courtney SE, Danhaive $O$, Carnielli VP et al. The Montreux definition of neonatal ARDS: biological and clinical background behind the description of a new entity. Lancet Respir Med. 2017; 5 (8): 657-666.

2. Ashbaugh DG, Boyd Bigelow D, Petty TL, Levine BE. Acute respiratory distress in adults. Lancet.1967; 290: 319-323.

3. Pepe PE, Potkin RT, Reus DH, Hudson LD, Carrico CJ. Clinical predictors of the adult respiratory distress syndrome. Am J Surg. 1982; 144: 124-130.

4. Murray JF, Matthay MA, Luce JM, Flick MR. An expanded definition of the adult respiratory distress syndrome. Am Rev Respir Dis. 1988; 138: 720-723.
5. Newth CJ, Stretton M, Deakers TW, Hammer J. Assessment of pulmonary function in the early phase of ARDS in pediatric patients. Pediatr Pulmonol. 1997; 23: 169-175.

6. Bernard GR, Artigas A, Brigham KL, Carlet J, Falke K, Hudson L et al. The American-European Consensus Conference on ARDS. Definitions, mechanisms, relevant outcomes, and clinical trial coordination. Am J Respir Crit Care Med. 1994; 149: 818-824.

7. ARDS Definition Task Force, Ranieri VM, Rubenfeld GD, Thompson $\mathrm{BT}$, Ferguson ND, Caldwell $\mathrm{E}$ et al. Acute respiratory distress syndrome: the Berlin definition. JAMA. 2012; 307: 2526-2533.

8. Ferguson ND, Fan E, Camporota L, Antonelli M, Anzueto A, Beale $\mathrm{R}$ et al. The Berlin definition of ARDS: an axpanded rationale, justification, and supplementary material. Intensive Care Med. 2012; 38: 1573-1582.

9. Khemani RG, Smith LS, Zimmerman JJ, Erickson S, for the Pediatric Acute Lung Injury Consensus Conference Group. Pediatric acute respiratory distress syndrome: definition, incidence, and epidemiology: proceedings from the Pediatric Acute Lung Injury Consensus Conference. Pediatric Critic Care Med. 2015; 16 (5 Suppl 1): S2340.

10. Tissières P, Ochoda A, Dunn-Siegrist I, Drifte G, Morales M, Pfister $R$ et al. Innate immune deficiency of extremely premature neonates can be reversed by interferon- $\gamma$. PLoS One 2012; 7: e32863.

11. Faix RG, Viscardi RM, DiPietro MA, Nicks JJ. Adult respiratory distress syndrome in full-term newborns. Pediatrics. 1989; 83: 971-976.

12. Changsong Wang, Libo Guo, Chunjie Chi, Xiaoyang Wang, Lei Guo, Weiwei Wang et al. Mechanical ventilation modes for respiratory distress syndrome in infants: a systematic review and network meta-analysis. Crit Care. 2015; 19: 108.

13. Randolph AG. Management of acute lung injury and acute respiratory distress syndrome in children. Crit Care Med. 2009; 37: 2448-2454

14. Günther A, Ruppert $C$, Schmidt R, Markat $P$, Grimminger $F$, Walmrath $D$ et al. Surfactant alteration and replacement in acute respiratory distress syndrome. Respir Res. 2001; 2: 353-364.

15. Touqui $L$, Arbibe L. A role for phospholipase A2 in ARDS pathogenesis. Mol Med Today. 1999; 5: 244-249.

16. De Luca D, Minucci A, Tripodi D, Piastra M, Pietrini D, Zuppi C et al. Role of distinct phospholipases A2 and their modulators in meconium aspiration syndrome in human neonates. Intensive Care Med. 2011; 37: 1158-1165.

17. Cogo PE, Facco M, Simonato M, De Luca D, De Terlizi F, Rizzotti $U$ et al. Pharmacokinetics and clinical predictors of surfactant redosing in respiratory distress syndrome. Intensive Care Med. 2011; 37: 510-517.

18. Raghavendran K, Willson D, Notter RH. Surfactant therapy for acute lung injury and acute respiratory distress syndrome. Crit Care Clin. 2011; 27: 525-559.

19. Zecca E, De Luca D, Baroni S, Vento G, Tiberi E, Romagnoli C et al. Bile acid-induced lung injury in newborn infants: a bronchoalveolar lavage fluid study. Pediatrics. 2008; 121: e146-149. 